\title{
Unextendible product bases and locally unconvertible bound entangled states
}

\author{
Sergey Bravy * \\ Institute for Quantum Information, \\ California Institute of Technology, \\ Pasadena, 91125 CA, USA.
}

(Dated: October 31, 2018)

\begin{abstract}
Mutual convertibility of bound entangled states under local quantum operations and classical communication (LOCC) is studied. We focus on states associated with unextendible product bases (UPB) in a system of three qubits. A complete classification of such UPBs is suggested. We prove that for any pair of UPBs $S$ and $T$ the associated bound entangled states $\rho_{S}$ and $\rho_{T}$ can not be converted to each other by LOCC, unless $S$ and $T$ coincide up to local unitaries. More specifically, there exists a finite precision $\epsilon(S, T)>0$ such that for any LOCC protocol mapping $\rho_{S}$ into a probabilistic ensemble $\left(p_{\alpha}, \rho_{\alpha}\right)$, the fidelity between $\rho_{T}$ and any possible final state $\rho_{\alpha}$ satisfies $F\left(\rho_{T}, \rho_{\alpha}\right) \leq 1-\epsilon(S, T)$.
\end{abstract}

PACS numbers: 03.65.Bz, 03.67.-a, 89.70.+c

\section{INTRODUCTION AND SUMMARY OF RESULTS}

One of the most challenging problems in the field of quantum information is to understand what transformations of multipartite entangled states can be realized by local quantum operations and classical communication (LOCC). This problem has many important applications such as construction of entanglement distillation protocols 1], quantum data hiding 2], and separability check [3]. The following question is of particular importance for all these applications. Given mixed states $\rho_{S}$ and $\rho_{T}$ of some fixed composite quantum system, is it possible to convert $\rho_{S}$ into $\rho_{T}$ by LOCC transformations? More specifically, we will be interested in stochastic approximate convertibility. Recall, that an output of a LOCC transformation applied to the state $\rho_{S}$ is a probabilistic ensemble $\left(p_{\alpha}, \rho_{\alpha}\right)$, where $\alpha$ is a classical random variable describing results of intermediate measurements and having a probability distribution $\left\{p_{\alpha}\right\}$. The state $\rho_{\alpha}$ is an output quantum state conditioned on $\alpha$. By stochastic convertibility we mean that $\rho_{\alpha}$ must coincide with $\rho_{T}$ for at least one outcome $\alpha$ (we assume that $p_{\alpha}>0$ for all $\alpha$ ). In other words, the conversion succeeds with some probability which is generally smaller than one. By stochastic approximate convertibility we mean that for any given precision $\epsilon>0$ the state $\rho_{S}$ can be stochastically converted into some state $\rho_{\epsilon}$ satisfying $F\left(\rho_{T}, \rho_{\epsilon}\right) \geq 1-\epsilon$, where $F$ is the fidelity. Note that the probability for the conversion to succeed may turn to zero as the precision $\epsilon$ turns to zero. If this is the case, we can not stochastically convert $\rho_{S}$ into $\rho_{T}$. The stochastic approximate convertibility seems to be the weakest one as far as one-copy-to-one-copy transformations are concerned.

For last several years the stochastic convertibility of

*Electronic address: serg@cs.caltech.edu multipartite pure states has been extensively studied. In the case of pure states LOCC transformation reduces to local filtering operations 12]. The question of convertibility by local filtering operations (LFO) can be investigated using the theory of normal forms and entanglement monotones which are invariant under LFO, see Ref. [4].

Conditions for stochastic convertibility of mixed states are less understood. It was conjectured in Ref. [5] that a two-qubit mixed state $\rho_{S}$ can be stochastically converted into a state $\rho_{T}$ iff the Bell-diagonal normal form of $\rho_{T}$ equals to convex sum of some separable state and the Bell-diagonal normal form of $\rho_{S}$.

In this paper we consider only mixed states of a very special form, namely bound entangled states associated with unextendible product bases in a system of three qubits. The notion of unextendible product basis (UPB) was originally introduced in Ref. 6]. In our setting UPB is a family of vectors $\left|S_{1}\right\rangle, \ldots,\left|S_{n}\right\rangle \in\left(\mathbb{C}^{2}\right)^{\otimes 3}$ such that

- Each vector $\left|S_{j}\right\rangle$ has a product form $\left|S_{j}\right\rangle=\left|A_{j}\right\rangle \otimes$ $\left|B_{j}\right\rangle \otimes\left|C_{j}\right\rangle$ for some one-qubit states $\left|A_{j}\right\rangle,\left|B_{j}\right\rangle$, and $\left|C_{j}\right\rangle$.

- $\left\langle S_{i} \mid S_{j}\right\rangle=\delta_{i j}$ for all $i$ and $j$,

- The orthogonal complement to the space spanned by the vectors $\left|S_{1}\right\rangle, \ldots,\left|S_{n}\right\rangle$ does not contain product vectors.

The vectors $\left|S_{j}\right\rangle$ are refered to as members of the UPB. If $S=\left\{\left|S_{j}\right\rangle\right\}$ is a UPB, the linear space spaned by its members will be denoted $\mathcal{H}_{S}$. A mixed state $\rho_{S}$ associated with a UPB $S$ is defined as a properly normalized projector onto an orthogonal complement of $\mathcal{H}_{S}$. In our setting it is

$$
\rho_{S}=\frac{1}{2^{3}-n}\left(I-\sum_{j=1}^{n}\left|S_{j}\right\rangle\left\langle S_{j}\right|\right) .
$$

It can not be a separable state, since its range does not contain product vectors. However, as was pointed out in 
Ref. [8], in the case of three qubits $\rho_{S}$ is separable with respect to any bipartite cut, for instance $A B C=A \cup B C$ (we will use letters $\mathrm{A}, \mathrm{B}$, and $\mathrm{C}$ to label the qubits). In particular it is not possible to distill some pure entanglement between any two qubits starting from many copies of $\rho_{S}$ and applying only LOCC transformations. For that reason $\rho_{S}$ is refered to as a bound entangled state.

In section [1] we build a complete classification of threequbit UPBs. It appears that each UPB has exactly four members. By local unitaries and permutations of the members we can bring any UPB into the following form:

$$
\begin{aligned}
& \left|S_{1}\right\rangle=|0\rangle \otimes|0\rangle \otimes|0\rangle, \\
& \left|S_{2}\right\rangle=|1\rangle \otimes|B\rangle \otimes|C\rangle, \\
& \left|S_{3}\right\rangle=|A\rangle \otimes|1\rangle \otimes\left|C^{\perp}\right\rangle, \\
& \left|S_{4}\right\rangle=\left|A^{\perp}\right\rangle \otimes\left|B^{\perp}\right\rangle \otimes|1\rangle,
\end{aligned}
$$

where

$$
\begin{aligned}
|A\rangle\langle A| & =(1 / 2)\left[I+\cos \left(\theta_{A}\right) \sigma^{z}+\sin \left(\theta_{A}\right) \sigma^{x}\right], \\
|B\rangle\langle B| & =(1 / 2)\left[I+\cos \left(\theta_{B}\right) \sigma^{z}+\sin \left(\theta_{B}\right) \sigma^{x}\right], \\
|C\rangle\langle C| & =(1 / 2)\left[I+\cos \left(\theta_{C}\right) \sigma^{z}+\sin \left(\theta_{C}\right) \sigma^{x}\right] .
\end{aligned}
$$

(Here and throught the paper we use a designation $\left|A^{\perp}\right\rangle$ for a state orthogonal to the state $|A\rangle$.) The family given by Eq. (2 3) specifies a UPB for an arbitrary triple $\theta_{A}, \theta_{B}, \theta_{C} \neq 0 \bmod \pi$. However some triples must be identified since the corresponding UPBs can be matched by local unitaries and permutation of the UPB's members (in this case the associated states are related by local unitaries). We show that the "fundamental" region of parameters corresponds to $\theta_{A}, \theta_{B}, \theta_{C} \in(0, \pi)$. Restricting ourselves to this region we count each UPB exactly one time.

Our main conclusion is that stochastic approximate LOCC conversion (in either direction) of states $\rho_{S}$ and $\rho_{T}$ associated with UPBs $S$ and $T$ is impossible, unless $\rho_{S}$ and $\rho_{T}$ are related by local unitaries. Following Ref. [7] we obtain the necessary conditions for LOCC convertibility finding the necessary conditions for convertibility by separable superoperators. So the statement which we have actually proved is following:

Theorem 1. Suppose $S$ and $T$ are three-qubit UPBs which are not related by local unitary operators and permutation of the members. Let $\rho_{S}$ and $\rho_{T}$ be the bound entangled states associated with $S$ and $T$. There exists a finite precision $\epsilon(S, T)>0$ such that for any separable completely positive superoperator $E$ satisfying $E\left(\rho_{S}\right) \neq 0$ one has

$$
F\left(\rho_{T}, \frac{E\left(\rho_{S}\right)}{\operatorname{Tr}\left[E\left(\rho_{S}\right)\right]}\right) \leq 1-\epsilon(S, T) .
$$

The paper is organized in the following way. In section 1 we build a classification of three-qubit UPBs and reveal some useful facts about them. The most important fact is Lemma 3 which says that members of UPB are the only product vectors in the spanning space of a UPB.
In section III we address stochastic (exact) convertibility of mixed states associated with UPBs. In section IV we examine a simplified version of the problem, namely approximate convertibility by local filtering operations. Section $\nabla$ contains a proof of Theorem [1] In Conclusion we summarize the results obtained in the paper and discuss possible application of our method to UPBs in a system $\mathbb{C}^{3} \otimes \mathbb{C}^{3}$.

\section{UNEXTENDIBLE PRODUCT BASES FOR THREE QUBITS}

In this section we put forward a complete classification of UPBs for a system of three qubits and prove some useful facts about them. The qubits will be refered to as A, B, C. Let $\left|S_{j}\right\rangle \in \mathbb{C}^{2} \otimes \mathbb{C}^{2} \otimes \mathbb{C}^{2}$ be members of UPB:

$$
\left|S_{j}\right\rangle=\left|A_{j}\right\rangle \otimes\left|B_{j}\right\rangle \otimes\left|C_{j}\right\rangle, \quad j=1, \ldots, n .
$$

By definition $\left\langle S_{i} \mid S_{j}\right\rangle=\delta_{i, j}$ for all $i$ and $j$. Let $\mathcal{H}_{S}$ be a linear $n$-dimensional space spanned by the basis vectors $S_{j}$ and $\rho_{S}$ be a density operator proportional to a projector onto orthogonal complement of $\mathcal{H}_{S}$, see Eq. (11). As an example consider a UPB "Shifts" suggested in Ref. [6]. Its members are

$$
\begin{aligned}
& \left|S_{1}\right\rangle=|0\rangle \otimes|0\rangle \otimes|0\rangle, \\
& \left|S_{2}\right\rangle=|1\rangle \otimes|-\rangle \otimes|+\rangle, \\
& \left|S_{3}\right\rangle=|+\rangle \otimes|1\rangle \otimes|-\rangle, \\
& \left|S_{4}\right\rangle=|-\rangle \otimes|+\rangle \otimes|1\rangle .
\end{aligned}
$$

(Here we use a standard notations $| \pm\rangle=2^{-1 / 2}(|0\rangle \pm|1\rangle)$.) If some product vector $|S\rangle=|A\rangle \otimes|B\rangle \otimes|C\rangle$ extends this basis, then one of vectors $|A\rangle,|B\rangle,|C\rangle$ must be orthogonal to at least two vectors from the family $\{|0\rangle,|1\rangle,|+\rangle,|-\rangle\}$, which is impossible.

An important characteristic of an UPB is its orthogonality graph, see Ref. [8]. For our purposes it will be convenient to introduce more general definition of an orthogonality graph.

Definition 1. Let $M=\left\{\left|\Psi_{1}\right\rangle, \ldots,\left|\Psi_{N}\right\rangle \in \mathcal{H}\right\}$ be a family of vectors in a finite-dimensional Hilbert space $\mathcal{H}$. An orthogonality graph $G=(V, E)$ of the family $M$ has a set of vertices $V=\{1, \ldots, N\}$ with one vertex assigned to each state $\left|\Psi_{j}\right\rangle$. A pair of vertices $(i, j)$ is an edge, $(i j) \in E$, iff $\left\langle\Psi_{i} \mid \Psi_{j}\right\rangle=0$.

For example, an orthogonality graph of any family of vectors in $\mathbb{C}^{2}$ is a collection of disjoint edges and isolated vertices. This definition will help us to prove the following statement.

\section{Lemma 1. Any three-qubit UPB has four members.}

Proof. First of all we note that the number of members $n$ can not be greater than 5 . Indeed, if $n \geq 6$, we have $\operatorname{Rk}\left(\rho_{S}\right) \leq 2$. From Ref. [9] we know that a PPT threequbit state with a rank two or one must be separable. 


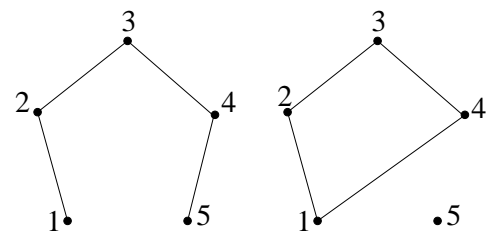

FIG. 1: Possible orthogonality graphs $G_{A}$ for the family $\left\{\left|A_{j}\right\rangle\right\}$.

But $\rho_{S}$ is not separable by definition. Let us prove that an existence of a UPB with five members also leads to a contradiction. Let $S$ be a such UPB with the members given by Eq. (4). Consider orthogonality graphs $G_{A}=\left(V, E_{A}\right), G_{B}=\left(V, E_{B}\right)$, and $G_{C}=\left(V, E_{C}\right)$ for the families $\left\{\left|A_{j}\right\rangle\right\},\left\{\left|B_{j}\right\rangle\right\}$, and $\left\{\left|C_{j}\right\rangle\right\}$ respectively. Here $V=\{1, \ldots, 5\}$ and

$$
\begin{aligned}
& (i, j) \in E_{A} \text { iff }\left\langle A_{i} \mid A_{j}\right\rangle=0, \\
& (i, j) \in E_{B} \text { iff }\left\langle B_{i} \mid B_{j}\right\rangle=0, \\
& (i, j) \in E_{C} \text { iff }\left\langle C_{i} \mid C_{j}\right\rangle=0,
\end{aligned}
$$

Orthogonality of the basis vectors $\left\langle S_{i} \mid S_{j}\right\rangle=\delta_{i, j}$ implies that any pair of vertices $(i, j)$ belongs to at least one of the sets $E_{A}, E_{B}, E_{C}$. Thus

$$
\left|E_{A}\right|+\left|E_{B}\right|+\left|E_{C}\right| \geq \frac{n(n-1)}{2}=10
$$

and at least one of the sets $E_{A}, E_{B}, E_{C}$ contains 4 or more edges. Assume that $\left|E_{A}\right| \geq 4$ and focus on the graph $G_{A}$. Observe that $G_{A}$ can not have a vertex with a valence 3 or greater. Indeed, if, say, $(1,2) \in E_{A}$, $(1,3) \in E_{A}$, and $(1,4) \in E_{A}$ then a state $\left|S_{6}\right\rangle=$ $\left|A_{1}\right\rangle \otimes\left|B_{1}^{\perp}\right\rangle \otimes\left|C_{5}^{\perp}\right\rangle$ extends a basis which is impossible. Besides, $G_{A}$ can not contain cycles with odd number of edges (this constraint comes from two-dimensional geometry). Summarizing, $G_{A}$ must match the following restrictions:

- there are at least 4 edges

- a valence of any vertex may be $0,1,2$ only

- there are no odd cycles

Up to permutations of the vertices there are only two graphs satisfying all the restrictions. They are shown on FIG. 1. For the graph on the left the basis can be extended by a state $\left|S_{6}\right\rangle=\left|A_{2}\right\rangle \otimes\left|B_{2}^{\perp}\right\rangle \otimes\left|C_{4}^{\perp}\right\rangle$. For the graph on the right the basis can be extended by a state $\left|S_{6}\right\rangle=\left|A_{5}^{\perp}\right\rangle \otimes\left|B_{5}\right\rangle \otimes\left|C_{5}\right\rangle$. Thus an assumption that UPB contains 5 members leads to a contradiction.

For our purposes the order of UPB members will not be important. Besides we would like to identify UPBs which can be matched by local unitary operators. Let us introduce the following equivalence relation:
Definition 2. UPBs $S$ and $S^{\prime}$ are equivalent, $S \sim S^{\prime}$, iff $\left|S_{j}\right\rangle=U_{A} \otimes U_{B} \otimes U_{C}\left|S_{\sigma(j)}^{\prime}\right\rangle, j=1,2,3,4$, for some unitary operators $U_{A}, U_{B}, U_{C}$ and some permutation $\sigma \in$ $S_{4}$.

We will see later (corollary to Lemma 3) that UPBs $S$ and $S^{\prime}$ are eqivalent iff the corresponding states $\rho_{S}$ and $\rho_{S^{\prime}}$ are related by local unitaries. Classification of UPB with 4 members is given by

Lemma 2. Each equivalence class of UPBs has a representative of the form

$$
\begin{aligned}
& \left|S_{1}\right\rangle=|0\rangle \otimes|0\rangle \otimes|0\rangle, \\
& \left|S_{2}\right\rangle=|1\rangle \otimes|B\rangle \otimes|C\rangle, \\
& \left|S_{3}\right\rangle=|A\rangle \otimes|1\rangle \otimes\left|C^{\perp}\right\rangle, \\
& \left|S_{4}\right\rangle=\left|A^{\perp}\right\rangle \otimes\left|B^{\perp}\right\rangle \otimes|1\rangle .
\end{aligned}
$$

For arbitrary $|A\rangle,|B\rangle,|C\rangle \notin\{|0\rangle,|1\rangle\}$ the set of states given by Eq. (5) is a UPB.

Proof. Suppose $S=\left\{\left|S_{j}\right\rangle\right\}$ is a UPB with four members given by Eq. (4). The unextendability implies that neither of the sets $\left\{\left|A_{j}\right\rangle\right\},\left\{\left|B_{j}\right\rangle\right\},\left\{\left|C_{j}\right\rangle\right\}$ can contain two equal states. Indeed, if, say, $\left|A_{1}\right\rangle=\left|A_{2}\right\rangle$, then a state $\left|S_{5}\right\rangle=\left|A_{1}^{\perp}\right\rangle \otimes\left|B_{3}^{\perp}\right\rangle \otimes\left|C_{4}^{\perp}\right\rangle$ extends the basis. One remains to take into account mutual orthogonality of the basis members. Applying local unitary operators we can make $\left|S_{1}\right\rangle=|0\rangle \otimes|0\rangle \otimes|0\rangle$. It follows that each of $\left|S_{2}\right\rangle$, $\left|S_{3}\right\rangle,\left|S_{4}\right\rangle$ contains at least one factor $|1\rangle$. Since neither of the sets $\left\{\left|A_{j}\right\rangle\right\},\left\{\left|B_{j}\right\rangle\right\},\left\{\left|C_{j}\right\rangle\right\}$ can contain two copy of $|1\rangle$, by appropriate permutation of the members $\left|S_{2}\right\rangle$, $\left|S_{3}\right\rangle,\left|S_{4}\right\rangle$ we can always make

$$
\begin{aligned}
& \left|S_{1}\right\rangle=|0\rangle \otimes|0\rangle \otimes|0\rangle, \\
& \left|S_{2}\right\rangle=|1\rangle \otimes\left|B_{2}\right\rangle \otimes\left|C_{2}\right\rangle, \\
& \left|S_{3}\right\rangle=\left|A_{3}\right\rangle \otimes|1\rangle \otimes\left|C_{3}\right\rangle, \\
& \left|S_{4}\right\rangle=\left|A_{4}\right\rangle \otimes\left|B_{4}\right\rangle \otimes|1\rangle,
\end{aligned}
$$

Orthogonality $\left\langle S_{2} \mid S_{3}\right\rangle=0$ translates into $\left\langle C_{2} \mid C_{3}\right\rangle=0$. Analogously, $\left\langle S_{2} \mid S_{4}\right\rangle=0$ implies that $\left\langle B_{2} \mid B_{4}\right\rangle=0$ and $\left\langle S_{3} \mid S_{4}\right\rangle=0$ gives us $\left\langle A_{3} \mid A_{4}\right\rangle=0$. Introducing $|A\rangle=\left|A_{3}\right\rangle,|B\rangle=\left|B_{2}\right\rangle$, and $|C\rangle=\left|C_{2}\right\rangle$ we arrive to a representation declared in Eq. (5).

Consider an orthogonal basis $\left\{\left|S_{j}\right\rangle\right\}$ as in Eq. (5) and suppose that $|A\rangle,|B\rangle,|C\rangle \notin\{|0\rangle,|1\rangle\}$. Assume that a state $|a\rangle \otimes|b\rangle \otimes|c\rangle$ extends the basis. It is orthogonal to each of $\left|S_{j}\right\rangle, j=1,2,3,4$ either on the qubit A, or on the qubit $\mathrm{B}$, or on the qubit $\mathrm{C}$. But each of the states $|a\rangle,|b\rangle$, $|c\rangle$ may provide orthogonality to at most one member of the family $\left\{\left|S_{j}\right\rangle\right\}$. Thus the basis is unextendible.

Let us count, how many real parameters we need to parameterize equivalence classes of UPBs. Applying local phase shifts to the qubits $\mathrm{A}, \mathrm{B}, \mathrm{C}$, we can always make

$$
\begin{aligned}
|A\rangle\langle A| & =(1 / 2)\left(I+\cos \left(\theta_{A}\right) \sigma^{z}+\sin \left(\theta_{A}\right) \sigma^{x}\right), \\
|B\rangle\langle B| & =(1 / 2)\left(I+\cos \left(\theta_{B}\right) \sigma^{z}+\sin \left(\theta_{B}\right) \sigma^{x}\right), \\
|C\rangle\langle C| & =(1 / 2)\left(I+\cos \left(\theta_{C}\right) \sigma^{z}+\sin \left(\theta_{C}\right) \sigma^{x}\right) .
\end{aligned}
$$


Different triples $\left(\theta_{A}, \theta_{B}, \theta_{C}\right)$ may still represent the same equivalence class. For example, applying $\sigma^{z}$ to the qubit A, we should identify $\theta_{A}$ and $-\theta_{A}$ (analogously, $\theta_{B} \equiv$ $-\theta_{B}$ and $\left.\theta_{C} \equiv-\theta_{C}\right)$. Thus all equivalence classes of UPBs are contained in the region $\theta_{A}, \theta_{B}, \theta_{C} \in(0, \pi)$. Although it is not necessary for the following discussion, in Appendix we prove that all triples $\left(\theta_{A}, \theta_{B}, \theta_{C}\right)$ inside this region represent different equivalence classes.

By definition, an orthogonal complement of a spanning space $\mathcal{H}_{S}$ of any UPB $S$ does not contain product vectors. The set of product vectors in the space $\mathcal{H}_{S}$ itself however is not empty. It always contains at least $n$ vectors - the members of UPB. Generally, the space $\mathcal{H}_{S}$ contains more than $n$ product vectors (some examples are given in Conclusion section). Amazingly, in the system of three qubits the members of UPB are the only product vectors in $\mathcal{H}_{S}$, which significantly simplify analysis of convertibility issues.

Lemma 3. Let $S$ be an arbitrary UPB for three qubits. The basis members $\left|S_{j}\right\rangle, j=1,2,3,4$ are the only product vectors in the spanning space $\mathcal{H}_{S}$.

Proof. Let $S=\left\{\left|S_{j}\right\rangle\right\}$ be a UPB with four members given by Eq. (5). We will prove more strong statement, namely, that the basis members $\left|S_{j}\right\rangle, j=1,2,3,4$ are the only product vectors in $\mathcal{H}_{S}$ with respect to any bipartite cut of three qubits. Consider for example a cut $A B C=$ $A \cup B C$. Suppose that for some $\left|\Psi_{A}\right\rangle \in \mathbb{C}^{2}$ and some $\left|\Psi_{B C}\right\rangle \in \mathbb{C}^{2} \otimes \mathbb{C}^{2}$ there exists a decomposition

$$
\left|\Psi_{A}\right\rangle \otimes\left|\Psi_{B C}\right\rangle=\sum_{j=1}^{4} \alpha_{j}\left|S_{j}\right\rangle, \quad \sum_{j=1}^{4}\left|\alpha_{j}\right|^{2}=1 .
$$

Tracing out the qubits $\mathrm{B}$ and $\mathrm{C}$ we get:

$$
\left|\Psi_{A}\right\rangle\left\langle\Psi_{A}\right|=\rho_{12}+\rho_{34},
$$

where

$$
\rho_{12}=\left(\begin{array}{cc}
\left|\alpha_{1}\right|^{2} & \alpha_{1} \bar{\alpha}_{2}\langle B \mid 0\rangle\langle C \mid 0\rangle \\
\bar{\alpha}_{1} \alpha_{2}\langle 0 \mid B\rangle\langle 0 \mid C\rangle & \left|\alpha_{2}\right|^{2}
\end{array}\right),
$$

and

$$
\rho_{34}=\left(\begin{array}{cc}
\left|\alpha_{3}\right|^{2} & \alpha_{3} \bar{\alpha}_{4}\left\langle B^{\perp} \mid 1\right\rangle\left\langle 1 \mid C^{\perp}\right\rangle \\
\bar{\alpha}_{3} \alpha_{4}\left\langle 1 \mid B^{\perp}\right\rangle\left\langle C^{\perp} \mid 1\right\rangle & \left|\alpha_{4}\right|^{2}
\end{array}\right) .
$$

Here $\rho_{12}$ is represented by its matrix elements in $\{|0\rangle,|1\rangle\}$ basis, while $\rho_{34}$ is represented by its matrix elements in $\left\{|A\rangle,\left|A^{\perp}\right\rangle\right\}$ basis. Since $\rho_{12}$ and $\rho_{34}$ are non-negative Hermitian operators, the equality in Eq. (9) is possible only if $\rho_{12}$ and $\rho_{34}$ are both proportional to the projector $\left|\Psi_{A}\right\rangle\left\langle\Psi_{A}\right|$. In particular it implies that $\operatorname{det} \rho_{12}=0$ and $\operatorname{det} \rho_{34}=0$. According to Lemma[2] the bases $\left\{|B\rangle,\left|B^{\perp}\right\rangle\right\}$ and $\left\{|C\rangle,\left|C^{\perp}\right\rangle\right\}$ do not coincide with the basis $\{|0\rangle,|1\rangle\}$. It means that the determinant

$$
\operatorname{det} \rho_{12}=\left|\alpha_{1} \alpha_{2}\right|^{2}\left(1-|\langle B \mid 0\rangle|^{2}|\langle C \mid 0\rangle|^{2}\right)
$$

equals zero only if $\alpha_{1} \alpha_{2}=0$. Analogously, $\operatorname{det} \rho_{34}=0$ only if $\alpha_{3} \alpha_{4}=0$. Since the basis $\left\{|A\rangle,\left|A^{\perp}\right\rangle\right\}$ does not coincide with $\{|0\rangle,|1\rangle\}$, the equality in Eq. (9) is possible only if at most one of the coefficients $\alpha_{1}, \alpha_{2}, \alpha_{3}, \alpha_{4}$ is nonzero. Thus the state $\left|\Psi_{A}\right\rangle \otimes\left|\Psi_{B C}\right\rangle$ coincides with one of the basis members.

Corollary 1. UPBs $S$ and $T$ are equivalent iff $\rho_{S}$ and $\rho_{T}$ are related by local unitaries.

Indeed, suppose that $\rho_{T}=U \rho_{S} U^{\dagger}$, for some unitary product operator $U=U_{A} \otimes U_{B} \otimes U_{C}$. Then $U \mathcal{H}_{S}=\mathcal{H}_{T}$ and thus vectors $U\left|S_{j}\right\rangle, j=1,2,3,4$ are some product vectors in $\mathcal{H}_{T}$. Lemma 3 says that these vectors are $\left|T_{j}\right\rangle$, up to some permutation. Thus $S \sim T$. The reverse statement is trivial.

Corollary 2. If UPBs $S$ and $T$ are not equivalent, they can not be converted to each other by local filtering operations.

To prove this corollary we will need two facts pointed out in Ref. 4], namely: 1) An orbit of any mixed state under LFO (or a closure of this orbit) contains a normal form, i.e. a state which has all one-particle marginals proportional to the identity. 2) Inside each orbit there is a unique (up to local unitaries) normal form. From the representation Eq. (5) it is clear that a state $\rho_{S}$ associated with an arbitrary UPB $S$ automatically comes in a normal form. Now the statement we need follows from the previous corollary and the two facts mentioned above. To ivestigate convertibility under general LOCC and approximate convertibility this results however is not strong enough. We will need one more lemma.

Lemma 4. Let $X=X_{A} \otimes X_{B} \otimes X_{C}$ be a non-degenerated (full rank) factorized 3-qubit operator such that

$$
X \cdot \mathcal{H}_{S}=\mathcal{H}_{T}
$$

for some UPBs $S$ and $T$. Then $S \sim T$ and $X$ is proportional to a unitary operator: $X=r U, r \in \mathbb{R}, U U^{\dagger}=I$.

Proof. Obviously, multiplying $X$ on factorized unitary operators on the left and on the right is equivalent to a choice of representatives in equivalence classes of $S$ and $T$. So we can assume that

\section{- $X_{A}, X_{B}$, and $X_{C}$ are Hermitian operators}

- Members of the UPB $S$ are given by Eq. (5).

Members of the UPB $T$ will be denoted as

$$
\begin{aligned}
& \left|T_{1}\right\rangle=\left|A_{1}\right\rangle \otimes\left|B_{1}\right\rangle \otimes\left|C_{1}\right\rangle, \\
& \left|T_{2}\right\rangle=\left|A_{2}\right\rangle \otimes\left|B_{2}\right\rangle \otimes\left|C_{2}\right\rangle, \\
& \left|T_{3}\right\rangle=\left|A_{3}\right\rangle \otimes\left|B_{3}\right\rangle \otimes\left|C_{3}\right\rangle, \\
& \left|T_{4}\right\rangle=\left|A_{4}\right\rangle \otimes\left|B_{4}\right\rangle \otimes\left|C_{4}\right\rangle .
\end{aligned}
$$

If $|\psi\rangle \in \mathcal{H}_{S}$ is a product vector, then its image $X|\psi\rangle \in$ $\mathcal{H}_{T}$ is also a product vector. Combining Lemma 3 and 
the fact that $X$ is non-degenerate operator, we conclude that

$$
X\left|S_{j}\right\rangle=x_{j}\left|T_{\sigma(j)}\right\rangle, \quad j=1,2,3,4,
$$

for some coefficients $x_{j} \neq 0$ and some permutation $\sigma \in$ $S_{4}$. Let us concentrate on the qubit A. From Eq. (11) we infer

$$
\begin{aligned}
X_{A}|0\rangle & =a_{1}\left|A_{\sigma(1)}\right\rangle, \\
X_{A}|1\rangle & =a_{2}\left|A_{\sigma(2)}\right\rangle, \\
X_{A}|A\rangle & =a_{3}\left|A_{\sigma(3)}\right\rangle, \\
X_{A}\left|A^{\perp}\right\rangle & =a_{4}\left|A_{\sigma(4)}\right\rangle,
\end{aligned}
$$

where $a_{j}$ are some non-zero coefficients. For each $j=$ $1,2,3,4$ choose some unital vector $\left|A_{j}^{\perp}\right\rangle$ orthogonal to $\left|A_{j}\right\rangle$. Taking into account the fact that $X_{A}$ is Hermitian and non-degenerated, we can rewrite Eq. (12) as

$$
\begin{aligned}
X_{A}\left|A_{\sigma(1)}^{\perp}\right\rangle & =b_{1}|1\rangle, \\
X_{A}\left|A_{\sigma(2)}^{\perp}\right\rangle & =b_{2}|0\rangle, \\
X_{A}\left|A_{\sigma(3)}^{\perp}\right\rangle & =b_{3}\left|A^{\perp}\right\rangle, \\
X_{A}\left|A_{\sigma(4)}^{\perp}\right\rangle & =b_{4}|A\rangle,
\end{aligned}
$$

for some non-zero coefficients $b_{j}$. Applying the classificaltion Lemma 2 to the UPB $T$ we conclude that the family $\left\{\left|A_{j}^{\perp}\right\rangle\right\}$ and the family $\left\{\left|A_{j}\right\rangle\right\}$ coincide up to permutation of the elements and some phase adjustment for each element. Moreover, from this Lemma we can learn that an orthogonality graph for the family $\left\{\left|A_{j}\right\rangle\right\}$ must be one of three graphs shown on FIG. 2.
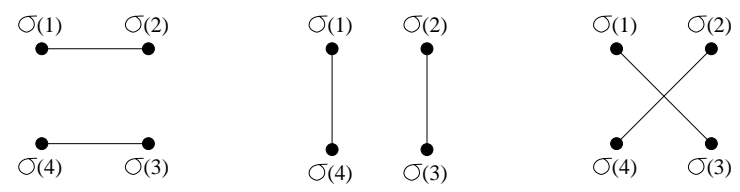

FIG. 2: Possible orthogonality graphs for the family $\left\{\left|A_{j}\right\rangle\right\}$.

(We choose an ordering of vertices which depends upon $\sigma$, since this ordering appears in Eq. (1213).) Whatever graph is chosen, the equations Eq. (12 13) guarantee that an operator $\left(X_{A}\right)^{4}$ is diagonal both in the basis $\{|0\rangle,|1\rangle\}$ and the basis $\left\{|A\rangle,\left|A^{\perp}\right\rangle\right\}$. By definition, these bases do not coincide. Thus $\left(X_{A}\right)^{4}$ is proportional to the identity, $\left(X_{A}\right)^{4}=x_{A} I$, for some coefficient $x_{A}$. Since $X_{A}$ is Hermitian, this is possible only if $X_{A}$ is proportional to a unitary operator. Applying the same arguments to the qubits $\mathrm{B}$ and $\mathrm{C}$, we conclude that $X$ is proportional to a unitary operator. Finally, Lemma 3 implies that $S \sim T$.

\section{EXACT CONVERTIBILITY OF BOUND ENTANGLED STATES}

Consider a pair of 3-qubit mixed states $\rho_{S}$ and $\rho_{T}$ associated with a pair of non-equivalent UPBs $S$ and $T$ respectively. Non-equivalence assumption implies that we can not convert $\rho_{S}$ into $\rho_{T}$ exactly by LFO, see the corollary to Lemma 3] Now we are in position to prove more strong statement, namely that $\rho_{S}$ can not be converted into $\rho_{T}$ exactly by a separable superoperators. To simplify notations, let us denote $\Omega_{f}$ a set of 3-qubit product operators, i.e.

$$
\Omega_{f}=\left\{X=X_{A} \otimes X_{B} \otimes X_{C}, \quad X_{A}, X_{B}, X_{C} \in \mathbb{L}\left(\mathbb{C}^{2}\right)\right\} .
$$

Lemma 5. Let $\left\{X_{l} \in \Omega_{f}\right\}_{l=1, \ldots, D}$ be a family of product operators such that $\sum_{l=1}^{D} X_{l}^{\dagger} X_{l} \leq I$. Suppose that for some UPBs $S, T$ and real number $p>0$ one has the equality

$$
\sum_{l=1}^{D} X_{l} \rho_{S} X_{l}^{\dagger}=p \rho_{T}
$$

Then the UPBs $S$ and $T$ are equivalent.

Remarks: 1) The factor $p$ can be regarded as a probability for conversion to succeed, since

$$
p=\operatorname{Tr}\left(\sum_{l=1}^{D} X_{l}^{\dagger} X_{l} \rho_{S}\right) \in[0,1] .
$$

2) Without loss of generality we can choose $D$ as a real dimension of a space of all superoperators on three qubits, which is $D=2^{13}$. Indeed, superoperators $E(\rho)=$ $\sum_{l} X_{l} \rho X_{l}^{\dagger}$ discussed in the lemma constitute a compact convex subset in the linear space of all superoperators on three qubits.

Proof. Denote

$$
\rho_{l}=X_{l} \rho_{S} X_{l}^{\dagger}
$$

Since $\rho_{l}$ is a positive semidefinite operator, the equality Eq. (14) tells us that

$$
\operatorname{Rg}\left(\rho_{l}\right) \subseteq \operatorname{Rg}\left(\rho_{T}\right), \quad l=1, \ldots, D,
$$

or, equivalently,

$$
X_{l} \cdot \operatorname{Rg}\left(\rho_{S}\right) \subseteq \operatorname{Rg}\left(\rho_{T}\right),
$$

where $\operatorname{Rg}(\rho)$ is a range of the operator $\rho$. Suppose that for some $l$ we have $\rho_{l} \neq 0$ and the operator $X_{l}$ is degenerated. Let us show that this assumption leads to a contradiction. By definition, $X_{l}$ has a product form, $X_{l}=X_{A} \otimes X_{B} \otimes X_{C}$, so at least one of the factors $X_{A}$, $X_{B}, X_{C}$ has a rank one. Consider the case $\operatorname{Rk}\left(X_{A}\right)=1$, i.e. $X_{A}=|\psi\rangle\langle\phi|$ for some vectors $|\psi\rangle,|\phi\rangle \in \mathbb{C}^{2}$. Then

$$
\begin{aligned}
\rho_{l} & =|\psi\rangle\langle\psi| \otimes \rho_{B C}, \\
\rho_{B C} & =X_{B} \otimes X_{C}\left\langle\phi\left|\rho_{S}\right| \phi\right\rangle X_{B}^{\dagger} \otimes X_{C}^{\dagger} .
\end{aligned}
$$

Since $\rho_{S}$ is a positive partial traspose (PPT) state, the same does $\rho_{l}$. Then from the equality Eq. (17) we infer that $\rho_{B C}$ is a two-qubit PPT state. But according to 
Peres criteria [10, 11] it implies that $\rho_{B C}$ is separable. Let us choose some product vector $\left|\psi_{B}\right\rangle \otimes\left|\psi_{C}\right\rangle$ from its range. Then a product vector $|\psi\rangle \otimes\left|\psi_{B}\right\rangle \otimes\left|\psi_{C}\right\rangle$ belongs to the range of $\rho_{l}$ and thus to the range of $\rho_{T}$, see Eq. (15). Since $\rho_{T}$ has no product vectors in its range, we conclude that for each $l$ either $X_{l}$ is non-degenerated or $\rho_{l}=0$.

Let us focus on some $l$ with $\rho_{l} \neq 0$. According to classification Lemma 22 the spaces $\operatorname{Rg}\left(\rho_{S}\right)$ and $\operatorname{Rg}\left(\rho_{T}\right)$ have the same dimension. Since $X_{l}$ is non-degenerated, Eq. (16) actually means that $X_{l} \cdot \operatorname{Rg}\left(\rho_{S}\right)=\operatorname{Rg}\left(\rho_{T}\right)$, or equivalently, that

$$
X_{l}^{\dagger} \cdot \mathcal{H}_{T}=\mathcal{H}_{S}
$$

According to Lemma 4 this is possible only if $S \sim T$.

Since stochastic LOCC transformations are described by separable superoperators, we have proved the following theorem:

Theorem 2. Let $S$ and $T$ be unextendible product bases for three qubits. Consider bound entangled states $\rho_{S}$ and $\rho_{T}$ associated with $S$ and $T$. If some LOCC transformation maps $\rho_{S}$ into $\rho_{T}$ with a non-zero probability, then the states $\rho_{S}$ and $\rho_{T}$ coincide up to local unitary transformation.

\section{APPROXIMATE CONVERTIBILITY: SIMPLIFIED SCENARIO}

Consider a three-qubit UPB $S=\left\{\left|S_{j}\right\rangle\right\}$ with the members given by Eq. (2). Let $T$ be another UPB which is not equivalent to $S$. In this section we will study a simplified version of the stochastic approximate convertibility problem. Namely, we will consider approximate convertibility by local filtering operations, i.e. transformations like

$$
\rho_{S} \rightarrow \rho=\frac{X \rho_{S} X^{\dagger}}{p_{S}[X]}, \quad p_{S}[X] \equiv \operatorname{Tr}\left(X \rho_{S} X^{\dagger}\right) .
$$

where $X \in \Omega_{f}$ is a product operator such that $p_{S}[X]>0$. Our goal is to prove that there exists a finite precision $\epsilon>$ 0 such that all states $\rho$ which can appear in Eq. (19) lie outside $\epsilon$-neighborhood of $\rho_{T}$ (we use fidelity to quantify the distance). Denote a set of achievable states $\rho$ as $M_{S}$ :

$$
M_{S}=\left\{\rho=\frac{X \rho_{S} X^{\dagger}}{p_{S}[X]}: X \in \Omega_{f}, \quad p_{S}[X]>0\right\} .
$$

The results of the previous section imply that $\rho_{T} \notin$ $M_{S}$. Note however that $M_{S}$ is not a compact set since $p_{S}[X]=0$ for some product operators $X$ (for instance, $p_{S}\left[\left|S_{j}\right\rangle\left\langle S_{j}\right|\right]=0$ for any member of the UPB). Thus there might exist a sequence of product operators $\left\{X_{n}\right\}_{n \geq 0}$, $p_{S}\left[X_{n}\right]>0$, such that the corresponding sequence $\rho\left(\bar{X}_{n}\right)$ converges to some operator $\rho$ which does not belong to
$M_{S}$. If $\rho=\rho_{T}$, it would imply that stochastic convertibility with an arbitrary small precision $\epsilon$ is possible (although the success probability may turn to zero as $\epsilon \rightarrow 0)$. Amazingly, this is not the case.

Lemma 6. Let $S$ be an arbitrary UPB. Consider a set of achievable states $M_{S}$ as in Eq. (20) and an arbitrary convergent operator sequence $\left\{Y_{n} \in M_{S}\right\}_{n \geq 0}$. Denote

$$
\hat{Y}=\lim _{n \rightarrow \infty} Y_{n} .
$$

Then either $\hat{Y} \in M_{S}$ or $\hat{Y}$ is separable.

Proof. Suppose that $\hat{Y} \notin M_{S}$. Obviously, in the definition of $M_{S}$ one suffices to consider normalized operators $X$. Let us agree that

$$
X=X_{A} \otimes X_{B} \otimes X_{C}, \quad\left\|X_{A}\right\|=\left\|X_{B}\right\|=\left\|X_{C}\right\|=1 .
$$

Let us choose an arbitrary sequence of normalized operators $\left\{X_{n} \in \Omega_{f}\right\}_{n \geq 0}, p_{S}\left[X_{n}\right]>0$, such that $Y_{n}=$ $X_{n} \rho_{S} X_{n}^{\dagger} / p_{S}\left[X_{n}\right]$. Without lose of generality we can assume that the sequence $\left\{X_{n}\right\}$ is also convergent (since $X_{n}$ are taken from a bounded manifold, we can always extract a convergent subsequence). Denote

$$
\hat{X}=\lim _{n \rightarrow \infty} X_{n}=\hat{X}_{A} \otimes \hat{X}_{B} \otimes \hat{X}_{C} .
$$

Note that $p_{S}[\hat{X}]=0$, since otherwise $\hat{Y} \in M_{S}$. It means that $\hat{X} \rho_{S} \hat{X}^{\dagger}=0$, or, equivalently, $\rho_{S} \hat{X}^{\dagger}=0$ which implies $\operatorname{Im}\left(\hat{X}^{\dagger}\right) \subseteq \operatorname{Ker}\left(\rho_{S}\right)$. Therefore

$$
\operatorname{Im}\left(\hat{X}_{A}^{\dagger}\right) \otimes \operatorname{Im}\left(\hat{X}_{B}^{\dagger}\right) \otimes \operatorname{Im}\left(\hat{X}_{C}^{\dagger}\right) \subseteq \mathcal{H}_{S} .
$$

But according to Lemma 3 the only product vectors in $\mathcal{H}_{S}$ are the members of $S$. Thus $\hat{X}=|a, b, c\rangle\left\langle S_{j}\right|$ for some $j \in[1,4]$ and some one-qubit normalized states $|a\rangle$, $|b\rangle,|c\rangle$ Without loss of generality, we can assume that $j=1$, i.e.

$$
\hat{X}_{A}=|a\rangle\left\langle 0\left|, \quad \hat{X}_{B}=\right| b\right\rangle\left\langle 0\left|, \quad \hat{X}_{C}=\right| c\right\rangle\langle 0|,
$$

(see Eq. (5)). The elements of the sequence $\left\{X_{n}\right\}_{n \geq 0}$ can always be written as

$$
\begin{aligned}
X_{n} & =X_{A, n} \otimes X_{B, n} \otimes X_{C, n}, \\
X_{A, n} & =\left|a_{n}\right\rangle\left\langle 0|+| \alpha_{n}\right\rangle\langle 1|, \\
X_{B, n} & =\left|b_{n}\right\rangle\left\langle 0|+| \beta_{n}\right\rangle\langle 1|, \\
X_{C, n} & =\left|c_{n}\right\rangle\left\langle 0|+| \gamma_{n}\right\rangle\langle 1|,
\end{aligned}
$$

where $\left|a_{n}\right\rangle,\left|b_{n}\right\rangle,\left|c_{n}\right\rangle$ and $\left|\alpha_{n}\right\rangle,\left|\beta_{n}\right\rangle,\left|\gamma_{n}\right\rangle$ are some onequbit states. The requirements Eq. (2122) translate into

$$
\lim _{n \rightarrow \infty}\left|a_{n}\right\rangle=|a\rangle, \quad \lim _{n \rightarrow \infty}\left|b_{n}\right\rangle=|b\rangle, \quad \lim _{n \rightarrow \infty}\left|c_{n}\right\rangle=|c\rangle,
$$

and

$$
\lim _{n \rightarrow \infty}\left\langle\alpha_{n} \mid \alpha_{n}\right\rangle=\lim _{n \rightarrow \infty}\left\langle\beta_{n} \mid \beta_{n}\right\rangle=\lim _{n \rightarrow \infty}\left\langle\gamma_{n} \mid \gamma_{n}\right\rangle=0 .
$$


Denote

$$
Y_{n}=\frac{X_{n} \rho_{S} X_{n}^{\dagger}}{p_{S}\left[X_{n}\right]}
$$

To compute a limit of $Y_{n}$ for $n \rightarrow \infty$ we can keep only the leading terms in the expression for $X_{n}$ (see a comment below), namely

$$
\begin{aligned}
X_{n} \approx & X_{n}^{(0)}+X_{n}^{(1)} \\
X_{n}^{(0)}= & \left|a_{n}, b_{n}, c_{n}\right\rangle\langle 0,0,0|, \\
X_{n}^{(1)}= & \left|\alpha_{n}, b_{n}, c_{n}\right\rangle\left\langle 1,0,0|+| a_{n}, \beta_{n}, c_{n}\right\rangle\langle 0,1,0| \\
& +\left|a_{n}, b_{n}, \gamma_{n}\right\rangle\langle 0,0,1| .
\end{aligned}
$$

Obviously, $X_{n}^{0}$ does not yield any contribution to $Y_{n}$, so that

$$
X_{n} \rho_{S} X_{n}^{\dagger} \approx X_{n}^{(1)} \rho_{S} X_{n}^{(1)^{\dagger}}
$$

Now we can substitute $a_{n}, b_{n}, c_{n}$ in the expression for $X_{n}^{(1)}$ by $a, b, c$ respectively, since we would like to keep only leading order terms. Using the list of the UPB's members Eq. (5) one can easily check that matrix elements $\left\langle 1,0,0\left|\rho_{S}\right| 0,1,0\right\rangle,\left\langle 1,0,0\left|\rho_{S}\right| 0,0,1\right\rangle$ (and their cyclic permutations) vanish. Therefore we arrive to

$$
\begin{array}{r}
X_{n}^{(1)} \rho_{S} X_{n}^{(1)^{\dagger}} \approx \\
\approx\left\langle 1,0,0\left|\rho_{S}\right| 1,0,0\right\rangle \cdot\left|\alpha_{n}, b, c\right\rangle\left\langle\alpha_{n}, b, c\right| \\
+\left\langle 0,1,0\left|\rho_{S}\right| 0,1,0\right\rangle \cdot\left|a, \beta_{n}, c\right\rangle\left\langle a, \beta_{n}, c\right| \\
+\left\langle 0,0,1\left|\rho_{S}\right| 0,0,1\right\rangle \cdot\left|a, b, \gamma_{n}\right\rangle\left\langle a, b, \gamma_{n}\right| .
\end{array}
$$

Note that this is a separable state and that the matrix elements

$$
\left\langle 1,0,0\left|\rho_{S}\right| 1,0,0\right\rangle,\left\langle 0,1,0\left|\rho_{S}\right| 0,1,0\right\rangle,\left\langle 0,0,1\left|\rho_{S}\right| 0,0,1\right\rangle,
$$

are strictly positive, since Lemma 3 tells us that the states $|1,0,0\rangle,|0,1,0\rangle$, and $|0,0,1\rangle$ do not belong to $\mathcal{H}_{S}$. It justifies that the leading terms in the expansion for $X_{n} \rho_{S} X_{n}^{\dagger}$ do not vanish and all terms we have disregarded are indeed small compared with the terms we keep. In particular,

$$
\begin{array}{r}
\operatorname{Tr}\left(X_{n}^{(1)} \rho_{S} X_{n}^{(1)^{\dagger}}\right) \approx \\
\left\langle 1,0,0\left|\rho_{S}\right| 1,0,0\right\rangle\left\langle\alpha_{n} \mid \alpha_{n}\right\rangle \\
+\left\langle 0,1,0\left|\rho_{S}\right| 0,1,0\right\rangle\left\langle\beta_{n} \mid \beta_{n}\right\rangle \\
+\left\langle 0,0,1\left|\rho_{S}\right| 0,0,1\right\rangle\left\langle\gamma_{n} \mid \gamma_{n}\right\rangle .
\end{array}
$$

We conclude that

$$
\hat{Y} \equiv \lim _{n \rightarrow \infty} Y_{n}=\lim _{n \rightarrow \infty} \frac{X_{n}^{(1)} \rho_{S} X_{n}^{(1)^{\dagger}}}{\operatorname{Tr}\left(X_{n}^{(1)} \rho_{S} X_{n}^{(1)^{\dagger}}\right)}
$$

where approximations Eq. (2627) should be substituted into nominator and denominator. But from Eq. (26) we infer that $\hat{Y}$ is separable.
Denote $\bar{M}$ a closure of the set $M$. It is a compact set. A fidelity $F\left(\rho, \rho_{T}\right)$ is a continuous function of a state $\rho$. According to Lemma [5] $F\left(\rho, \rho_{T}\right)<1$ for all $\rho \in M_{S}$. Lemma 6] implies that $F\left(\rho, \rho_{T}\right)<1$ even for all $\rho \in \overline{M_{S}}$. Thus there exists a finite precision $\epsilon>0$ such that

$$
F\left(\rho, \rho_{T}\right) \leq 1-\epsilon, \quad \text { for any } \rho \in M_{S} .
$$

Of course the precision $\epsilon$ may depend upon $S$ and $T$, in particular $\epsilon \rightarrow 0$ as $S$ turns to $T$.

\section{APPROXIMATE CONVERTIBILITY IN A GENERAL CASE}

We now are prepared to analyse approximate convertibility by arbitrary completely positive separable superoperators. Such operators correspond to probabilistic mixtures of LFO, so we can exploit the results of section [V] A proof of Theorem 1 is contained in the following lemma.

Lemma 7. Let $S$ and $T$ be non-equivalent UPBs. Suppose $\left\{X_{l} \in \Omega_{f}\right\}_{l=1, \ldots, D}$ is a family of product operators such that $p_{S}\left[X_{l}\right] \equiv \operatorname{Tr}\left(X_{l} \rho_{S} X_{l}^{\dagger}\right)>0$ for all $l$. Denote $p_{S}=\sum_{l=1}^{D} p_{S}\left[X_{l}\right]$. There exist a finite precision $\epsilon=\epsilon(S, T)>0$ depending upon $S$ and $T$ only, such that

$$
F\left(\rho_{T}, \frac{1}{p_{S}} \sum_{l=1}^{D} X_{l} \rho_{S} X_{l}^{\dagger}\right) \leq 1-\epsilon(S, T) .
$$

Proof. We start from introducing an auxiliary proximity measure between $\rho_{T}$ and the final state. Let $\left|T_{j}\right\rangle$, $j=1,2,3,4$ be the members of the UPB $T$. Consider a functional

$$
f_{T}(\rho)=\sum_{j=1}^{4}\left\langle T_{j}|\rho| T_{j}\right\rangle
$$

It is clear that

$$
f_{T}(\rho)=0 \quad \text { iff } \quad \operatorname{Rg}(\rho) \subseteq \operatorname{Rg}\left(\rho_{T}\right) .
$$

Thus the equality $f_{T}(\rho)=0$ is necessary for $\rho=\rho_{T}$. Define $\rho$ as

$$
\rho=\frac{1}{p_{S}} \sum_{l=1}^{D} X_{l} \rho_{S} X_{l}^{\dagger}
$$

Let us first prove that there exist a finite precision $\delta>0$ depending upon $S$ and $T$ only, such that

$$
f_{T}(\rho) \geq \delta .
$$

Using linearity of $f_{T}$ we can write

$$
\begin{aligned}
f_{T}(\rho) & =\sum_{l=1}^{D} \frac{p_{S}\left[X_{l}\right]}{p_{S}} f_{T}\left(Y_{l}\right), \\
Y_{l} & \equiv \frac{X_{l} \rho_{S} X_{l}^{\dagger}}{p_{S}\left[X_{l}\right]} \in M_{S},
\end{aligned}
$$


see Eq. (20). Using the same arguments as in the proof of Lemma 5 one can easily show that an inclusion $\operatorname{Rg}\left(Y_{l}\right) \subseteq \operatorname{Rg}\left(\rho_{T}\right)$ is impossible for any $Y_{l} \in M_{S}$. From Eq. (31) we infer that $f_{T}\left(Y_{l}\right)>0$ for any $Y_{l} \in M_{S}$. Moreover, $f_{T}(Y)>0$ for any $Y$ belonging to a closure of the set $M_{S}$. Indeed, Lemma 6 tells us that such $Y$ either belongs to $M_{S}$ or is separable. In the latter case the inclusion $\operatorname{Rg}(Y) \subseteq \operatorname{Rg}\left(\rho_{T}\right)$ is impossible, since $\operatorname{Rg}\left(\rho_{T}\right)$ is free of product vectors. Summarizing, there exists a finite precision $\delta$, such that

$$
f_{T}(Y) \geq \delta, \quad \text { for any } \quad Y \in M_{S} .
$$

Returning to Eq. (33) we immediately get the estimate Eq. (32).

To conclude the proof we need to turn inequality Eq. (32) into an upper bound on fidelity $F\left(\rho_{T}, \rho\right)$. It can be done as follows. We start from the standard definition of fidelity:

$$
F\left(\rho_{T}, \rho\right)=\operatorname{Tr}\left[\left(\sqrt{\rho_{T}} \rho \sqrt{\rho_{T}}\right)^{\frac{1}{2}}\right] .
$$

Introduce orthogonal projectors $P_{T}$ and $P_{T}^{\perp}$ onto the spanning space $\mathcal{H}_{T}$ and its orthogonal complement respectively. By definition, $P_{T}+P_{T}^{\perp}=I$. Then $\rho_{T}=$ $(1 / 4) P_{T}^{\perp}$ and thus

$$
F\left(\rho_{T}, \rho\right)=\frac{1}{2} \operatorname{Tr} \sqrt{P_{T}^{\perp} \rho P_{T}^{\perp}} .
$$

Note that $f_{T}(\rho)=\operatorname{Tr}\left(\rho P_{T}\right)$. From an identity

$$
\operatorname{Tr}\left(P_{T}^{\perp} \rho P_{T}^{\perp}\right)+\operatorname{Tr}\left(P_{T} \rho P_{T}\right)=1
$$

and from the estimate Eq. (32) we get

$$
\operatorname{Tr}\left(P_{T}^{\perp} \rho P_{T}^{\perp}\right) \leq 1-\delta .
$$

Taking into account that the operator $P_{T}^{\perp} \rho P_{T}^{\perp}$ has a rank at most four and applying Cauchy-Schwarz inequality we arrive to

$$
\operatorname{Tr} \sqrt{P_{T}^{\perp} \rho P_{T}^{\perp}} \leq \sqrt{4 \operatorname{Tr}\left(P_{T}^{\perp} \rho P_{T}^{\perp}\right)} \leq 2 \sqrt{1-\delta} .
$$

Substituting it to Eq. (36) gives us

$$
F\left(\rho_{T}, \rho\right) \leq \sqrt{1-\delta} \leq 1-\frac{\delta}{2} .
$$

We have proved the lemma.

\section{CONCLUSION}

We have studied local convertibility of three-qubit mixed states associated with unextendible product bases. A complete classification of three-qubit UPBs is suggested. This family of UPBs is shown to have some nice mathematical properties which allow to investigate convertibility question completely. We proved that for any non-equivalent UPBs $S$ and $T$ the stochastic approximate conversion of the associated states $\rho_{S}$ and $\rho_{T}$ is impossible.

It would be interesting to apply our method to UPBs in some other low-dimension systems. The system of two qutrits $\mathbb{C}^{3} \otimes \mathbb{C}^{3}$ is of particular interest, since for this system a complete UPBs classification has been already found in Ref. 8]. It is known that all two-qutrit UPBs are characterized by the same orthogonality graphs and consist of five members. Unfortunately a set of product vectors in a spaning space of a UPB is generally larger than a set of the UPB's members. We have checked it for two particular two-qutrit UPBs, called in Ref. [6] as "Tiles" and "Pyramid". Using the designations of this reference, the extra product vectors in the spaning spaces of "Pyramid" and "Tiles" respectively can be written as

$|0\rangle \otimes|0\rangle \quad$ and $\quad \frac{1}{9}(2|0\rangle-|1\rangle+2|2\rangle) \otimes(2|0\rangle-|1\rangle+2|2\rangle)$.

Exploiting the symmetry of these particular UPBs one can show that there are exactly six product vectors in their spaning spaces. However the presence of extra product vectors as well as three-dimensional geometry makes the convertibility analysis very complicated.

\section{Acknowledgments}

We would like to thank Guifre Vidal for supporting ideas which significantly simplify the proof in Section III We also acknowledge useful conversations with Patrick Hayden, Alexei Kitaev, and Federico Spedalieri.

\section{APPENDIX}

In this section we prove that all UPBs given by Eq. (56) with parameters $\theta_{A}, \theta_{B}, \theta_{C} \in(0, \pi)$ are not equivalent to each other in the sense of Definition 2 Consider UPBs $S=\left\{\left|S_{j}\right\rangle\right\}$ and $S^{\prime}=\left\{\left|S_{j}^{\prime}\right\rangle\right\}$ with the members

$$
\begin{aligned}
& \left|S_{1}\right\rangle=|0\rangle \otimes|0\rangle \otimes|0\rangle, \\
& \left|S_{2}\right\rangle=|1\rangle \otimes|B\rangle \otimes|C\rangle, \\
& \left|S_{3}\right\rangle=|A\rangle \otimes|1\rangle \otimes\left|C^{\perp}\right\rangle, \\
& \left|S_{4}\right\rangle=\left|A^{\perp}\right\rangle \otimes\left|B^{\perp}\right\rangle \otimes|1\rangle,
\end{aligned}
$$

and

$$
\begin{aligned}
\left|S_{1}^{\prime}\right\rangle & =|0\rangle \otimes|0\rangle \otimes|0\rangle, \\
\left|S_{2}^{\prime}\right\rangle & =|1\rangle \otimes\left|B^{\prime}\right\rangle \otimes\left|C^{\prime}\right\rangle, \\
\left|S_{3}^{\prime}\right\rangle & =\left|A^{\prime}\right\rangle \otimes|1\rangle \otimes\left|C^{\prime \perp}\right\rangle, \\
\left|S_{4}^{\prime}\right\rangle & =\left|A^{\prime \perp}\right\rangle \otimes\left|B^{\prime \perp}\right\rangle \otimes|1\rangle .
\end{aligned}
$$


Since the overall phase of the vectors is not important, the parameterization Eq. (3) is equivalent to

$$
\begin{aligned}
|A\rangle & =\cos \left(\theta_{A} / 2\right)|0\rangle+\sin \left(\theta_{A} / 2\right)|1\rangle, \\
|B\rangle & =\cos \left(\theta_{B} / 2\right)|0\rangle+\sin \left(\theta_{B} / 2\right)|1\rangle, \\
|C\rangle & =\cos \left(\theta_{C} / 2\right)|0\rangle+\sin \left(\theta_{C} / 2\right)|1\rangle,
\end{aligned}
$$

and

$$
\begin{aligned}
\left|A^{\prime}\right\rangle & =\cos \left(\theta_{A}^{\prime} / 2\right)|0\rangle+\sin \left(\theta_{A}^{\prime} / 2\right)|1\rangle, \\
\left|B^{\prime}\right\rangle & =\cos \left(\theta_{B}^{\prime} / 2\right)|0\rangle+\sin \left(\theta_{B}^{\prime} / 2\right)|1\rangle, \\
\left|C^{\prime}\right\rangle & =\cos \left(\theta_{C}^{\prime} / 2\right)|0\rangle+\sin \left(\theta_{C}^{\prime} / 2\right)|1\rangle .
\end{aligned}
$$

Suppose that

$$
U_{A} \otimes U_{B} \otimes U_{C}\left|S_{j}\right\rangle=\left|S_{\sigma(j)^{\prime}}\right\rangle
$$

for some one-qubit unitary operators $U_{A}, U_{B}, U_{C}$ and permutation $\sigma \in S_{4}$. If $\sigma(1)=1$ then orthogonality im- plies $\sigma(j)=j$ for all $j$. It means that $|\langle 0 \mid A\rangle|=\left|\left\langle 0 \mid A^{\prime}\right\rangle\right|$, $|\langle 0 \mid B\rangle|=\left|\left\langle 0 \mid B^{\prime}\right\rangle\right|$, and $|\langle 0 \mid C\rangle|=\left|\left\langle 0 \mid C^{\prime}\right\rangle\right|$. From Eq. A.3. and Eq. A.4 we have $\left|\cos \left(\theta_{A} / 2\right)\right|=\left|\cos \left(\theta_{A}^{\prime} / 2\right)\right|$. Since $\theta_{A}, \theta_{A}^{\prime} \in(0, \pi)$ we conclude that $\theta_{A}^{\prime}=\theta_{A}$. Analogously one gets $\theta_{B}^{\prime}=\theta_{B}$ and $\theta_{C}^{\prime}=\theta_{C}$.

If $\sigma(2)=1$ then orthogonality implies $\sigma(2)=1$, $\sigma(3)=4$, and $\sigma(4)=3$. It means that $|\langle 0 \mid A\rangle|=$ $\left|\left\langle 1 \mid A^{\prime \perp}\right\rangle\right|,|\langle 0 \mid B\rangle|=\left|\left\langle 0 \mid B^{\prime}\right\rangle\right|$, and $|\langle 0 \mid C\rangle|=\left|\left\langle 0 \mid C^{\prime}\right\rangle\right|$. Thus $\left(\theta_{A}, \theta_{B}, \theta_{C}\right)$ and $\left(\theta_{A}^{\prime}, \theta_{B}^{\prime}, \theta_{C}^{\prime}\right)$ coincide.

If $\sigma(3)=1$ then orthogonality implies $\sigma(1)=3$, $\sigma(2)=4$, and $\sigma(4)=2$. It means that $|\langle 0 \mid A\rangle|=\left|\left\langle 0 \mid A^{\prime}\right\rangle\right|$, $|\langle 0 \mid B\rangle|=\left|\left\langle 1 \mid B^{\prime \perp}\right\rangle\right|$, and $\left|\left\langle 0 \mid C^{\perp}\right\rangle\right|=\left|\left\langle 0 \mid C^{\perp}\right\rangle\right|$. Thus $\left(\theta_{A}, \theta_{B}, \theta_{C}\right)$ and $\left(\theta_{A}^{\prime}, \theta_{B}^{\prime}, \theta_{C}^{\prime}\right)$ coincide.

Finally, if $\sigma(4)=1$ then orthogonality implies $\sigma(1)=$ 4, $\sigma(2)=3$, and $\sigma(3)=2$. It means that $\left|\left\langle 0 \mid A^{\perp}\right\rangle\right|=$ $\left|\left\langle 0 \mid A^{\prime \perp}\right\rangle\right|,\left|\left\langle 0 \mid B^{\perp}\right\rangle\right|=\left|\left\langle 0 \mid B^{\perp}\right\rangle\right|$, and $|\langle 0 \mid C\rangle|=\left|\left\langle 1 \mid C^{\prime \perp}\right\rangle\right|$. Thus $\left(\theta_{A}, \theta_{B}, \theta_{C}\right)$ and $\left(\theta_{A}^{\prime}, \theta_{B}^{\prime}, \theta_{C}^{\prime}\right)$ coincide.
[1] C. H. Bennett, G. Brassard, S. Popescu, B. Schumacher, J. A. Smolin, and W. K. Wootters, Phys. Rev. Lett. 76, 722 (1996).

[2] D. DiVincenzo, D. Leung, and B. Terhal, IEEE Trans. Inf. Theory 48, 580 (2002).

[3] R. F. Werner, Phys. Rev. A 40, 4277 (1989).

[4] F. Verstraete, J. Dehaene, and B. De Moor, Phys. Rev. A 64, 032111 (2001).

[5] F. Verstraete, J. Dehaene, and B. De Moor, Phys. Rev. A 65, 032308 (2002).

[6] C. Bennett, D. DiVincenzo, T. Mor, P. Shor, J. Smolin, and B. Terhal, Phys. Rev. Lett. 82, 5385 (1999).
[7] E. M. Rains, e-print quant-ph/9707002 (1997).

[8] D. DiVincenzo, T. Mor, P. Shor, J. Smolin, and B. Terhal, Comm. Math. Phys. 238, 379 (2003).

[9] S. Karnas and M. Lewenstein, e-print quant-ph/0102115 (2001).

[10] A. Peres, Phys.Rev.Lett. 77, 1413 (1996).

[11] M. Horodecki, P. Horodecki, and R. Horodecki, e-print quant-ph/9605038 (1996).

[12] By local filtering operations we mean a transformation $\rho \rightarrow X \rho X^{\dagger}$, where $X=X_{A} \otimes X_{B} \otimes \cdots$ is an arbitrary product operator. 